\title{
Review on Biobased Mesoporous Material and Their Application in Waste Water Treatment
}

\author{
Priti Malhotra $^{1 *}$, Arti Jaina and Rekha Kathal ${ }^{2}$ \\ ${ }^{1}$ Department of Chemistry, Daulat ram College, University of Delhi, India \\ ${ }^{2}$ Department of Botany, Daulat ram College, University of Delhi, India
}

Submission: April 13, 2017; Published: May 24, 2017

*Corresponding author: Priti Malhotra, Department of Chemistry, Daulat ram College, University of Delhi, New Delhi 110007, India, Email: pritimalhotra21@ gmail.com

\begin{abstract}
Mesoporous material derived from bio based carbonaceous polysaccharides have recently attracted a lot if attention and have led to several innovations. A large number of environmentally friendly biobased adsorbents have been successfully prepared by the conversion of starch and other polysaccharide containing biowastes to carbonaceous mesoporous composite material. Owing to their great potential in remedial and bio applications, various methods of mesoporous material have been designed and modifications have been proposed for their controlled synthesis from various natural precursors. They have gradually emerged as efficient multifunctional tools in biosciences and have been widely explored for a broad range of applications. Since bioderived material display unique properties for their selective adsorption capacity, they have been potentially utilized in absorbing the contaminants of waste water which contains heavy metals and organic dyes along with other toxins. This article focuses on a concise overview of mesoporous material which are designed and derived from carbonaceous precursors and find outstanding applications in absorbing heavy metals and organic dyes present in waste water.
\end{abstract}

Keywords: Bio based materials; Mesoporous; Water purification; Environmental sustainability

\section{Introduction}

Over the recent past a wide range of composite material have been developed which have proved to be versatile absorbents, catalysts, sensors and separating agents. Among these, mesoporous substances have received widespread interest due to their extremely high surface areas combined with large, uniform and well defined pore sizes. When long chain surfactant molecules were employed as the structure directing agent during the synthesis of these highly ordered materials, their structure, composition and pore size could be tailored. It could be achieved by varying reaction pathways, reactant stoichiometry, reaction conditions, and fictionalization techniques. Variation in synthesis strategies can yield pore sizes from 2 to $10 \mathrm{~nm}$ also changing the pore topologies from cubic to hexagon in 2D or 3D and surface groups like silanol in mesoporous silica furnish tunable pore size and volume and cater to a wide range of applications.

An holistic approach to the vast applications of mesoporous structures lies in their preparation from renewable resources whose advantages range from economic viability to environment sustainability. Benign resources like biomass contain high percentage of and are used for raising mesoporous carbon which not only replaces the conventional activated carbon and its applications but also provide potential templates for mesoporous surfaces.

The use of more ecologically sustainable and more economical porogens for developing mesoporous silica are driven by following specifications:

i. Environmentally safe synthesis and production processes of the structuring agent

ii. Expansion of mesoporous material ,including the removal of templates following the path of atom economy

iii. Promoting safe methods for the degradation of structuring agent. Based on these specifications many new surfactants with lower toxicity and increased biodegradability were developed and a large number of studies were carried out on the environmentally benign applications of both non ordered and ordered mesoporous silica.

The powerful role of mesoporous material as efficient absorbents particularly of heavy metals (cations, oxyanions and radionuclides), toxic organic species and even gasses (e.g volatile organic compounds )has been well recognized [1-3] Mesoporous 
materials have also proved to outperform the classical silica materials as well as commercially used absorbents: activated carbon. Metal ion adsorption on the surface of these substances is one of the most intriguing researches and has gained significance due to waste water treatment which is an essential exercise for environment remediation.

\section{Discussion}

Nature is skilful at the self meeting of organic polymers (e.g. polysaccharides) in a wide range of structural and stowing roles in higher plants. Many biomaterials are organized from nanoscale to macroscopic scale to give hierarchical materials that are shaped with complex forms including spirals, spheroids and skeletons. The delicacy of Nature's approach may be exemplified with reverence to the glycosidic bond type. The reader need only to contrast the physiological roles of cellulose (polymeric b (1-4)-D-glucose)and amylose (polymeric a(1-4)-D-glucose) in higher plants. The former provides dimensional constancy to cell walls whilst the concluding acts as a energy vehicle in plant metabolism. Therefore:

a. Can Nature help in the preparation of mesoporous materials from agricultural waste?

b. Are preformed porous constructions accessible and reachable to the materials chemist for the generation of novel mesoporous carbonaceous materials?

We have found that polysaccharides can be manipulated in the aqueous phase to generate highly mesoscopic gels, the construction of which may be maintained in a final dried productvia a solvent exchange/drying procedure. Gel ordering of then polysaccharide upon recrystallisation from the gelation step, promotes the formation of an amphiphilic network, where hydrogen bond formation amid associated polysaccharide chains is the key to phase separation and subsequent gel mesophase development.

Polysaccharides have thermal properties which present a short melting stage (e.g. hydrogen bond network breakdown) followed by a main sharp endothermic decomposition. Potentially, this thermal event could destroy the mesopore polysaccharide (e.g. starch) network. In this regard, discoverey is done on the use of a strong Bronsted acid catalysis (e.g. p-toluene sulfonic acid) promotes non-acidic polysaccharide (e.g. starch) dehydration at temperatures below the sharp endothermic decomposition, facilitating cross linking and fixing of mesoporous network.

Starch is poised of both [linear a(1-4)] amylose and [branched $\mathrm{a}(1-4)$ and $\mathrm{a}(1-6)]$ amylopectin, which self assemble inside the starch granule to generate a semi-crystalline polysaccharide composite, presenting crystalline scopes in the mesopore size range (i.e. 4-5 -nm)-credited to spatial gaps in the crystalline amylopectin macromolecular structure, normally occupied bby the amorphous amylose content of the granule [4].
This led in part to the initial decision that gelatinisation of the starch granule ensued in leaching of the amorphous component to leave unbroken the remaining crystalline component (i.e.amylopectin) with potentially a residual preformed mesoporosity. However, systematic studies of synthetic amylose/ amylopectin mixtures revealed a different picture [5].

Mesoporosity showed a strong negative linear reliance of the amylopectin content of the gel mixture. These results specified that the key component to highly mesoporous starch was a minimal amylose content, whilst materials with advanced polymer ordering could be organized by the use of increased amylopectin content. Further delicate management of the morphology and textural properties of high amylase starch based porous materials were performed, by (microwave) gelatinisation temperature as a control vector, and pointed to the option of producing materials from the same polysaccharide with contrary tune able textural properties through the generation of differing meta stable gel states.

Amylose in principle displays many resemblances to other commonly found polysaccharides; it is a linear polysaccharide, unlike the atypical amylopectin, which holds a branched Super structure. Consequently, these results elevated an initially interesting empirical research question: "Can dissimilar polysaccharides of a single chain linear (e.g. helical forming) type be exploited to produce different mesoporous (metastable) gel states, and therefore porous polysaccharide-derived materials (PPDMs) owning differing textural properties?'. In the Same background, if the answer to this question was positive, it might be possible that the resulting PPDMs could be converted into mesoporous carbonaceous materials, showing tune able properties, potentially reflective of dissimilar glycosidic linkages, monomer structure and polysaccharide functionality.

\section{Metal Ion Adsorption}

The adsorption of metal ions is one of the most investigated research areas because of its importance in waste water treatment and purification processes. Various mesoporous materials have been used for this application. We will not discuss all these individual cases in detail, some of them are reviewed by Walcarius et al. [2] in 2010. We summarize some representative papers on this matter in Adsorption of organic dyes: In ancient times, fabrics have been dyed with natural color extracts obtained from plants, animals, and minerals. Now a day, things began to change with the synthesis of cheaper, easy to put on to the fabrics, brighter, and color fastening synthetic dyes. Human beings are becoming more and more dependent on these dyes for their simple house hold chores as well as other purposes. Although, these dyes are synthesized using the chemicals which are often carcinogenic, highly toxic, or even explosive. For eg., the chemical aniline, used as reactant to synthesis azo dyes is highly flammable, dangerous to deal and is considered poisonous (gives off carcinogenic amines). Practically every commercial 
dye process includes a solution of a dye in water, in which the fabrics are dipped or washed. After dying a lot of fabric, it's convenient to dump the used water that is dye effluent, than to recycle that water in the factory. So dye industries across the world are discharging millions of tons of dye effluent into rivers and water sources [6-9].

Crystal violet, one of the widely used cationic dyes in numerous industries can easily enter and accumulate into living organisms. Therefore, it is today's need to develop environmental friendly, low cost process for discovering heavy metals and dyes from industrial effluents. Heavy metal ions and organic dyes can be removed from waste water solutions via modified polymeric materials through reactive functional groups on their surfaces. Methylene blue with molecular formula: C16H18CIN3S3H2O is a toxic heterocyclic aromatic molecule, may produce problems if inhaled, swallowed or made in contact with skin or eye. Methylene blue is used in various studies; hence during its use a substantial amount of dye swamped with wastewater into the oceans and became hazardous for the aquatic lives. Therefore, decolorization and quenching of dyes is important aspects of wastewater remediation before discharge.

\section{Conclusion}

Soft template goes pathways for the production of porous and adding a surfactant containing solution and flexibility in synthesis conditions can lead to mesopore size and regulate its morphology making it suitable and efficient absorbing surface. The large surface area afforded by the presence of mesopores provides short diffusion lengths and a large contact area for potential adsorption. The drive towards bio-economy further boosts the concept and need to synthesize this novel family of mesoporous polysaccharide based carbonaceous material whose efficient adsorption characteristics can be universally applied for adsorption of contaminants like heavy metals and organic dyes from waste water streams.

\section{Acknowledgement}

Authors acknowledge university of Delhi for providing financial assistance for star innovation project DRC-05.

\section{Conflicts of Interest}

The authors declare no conflict of interest.

\section{References}

1. Wang PY, Liu X, Yang J, Yang Y, Zhang L, et al. (2009) Chirally functionalized mesoporous organosilicas with built-in BINAP ligand for asymmetric catalysis. J Mater Chem 19: 8009-8014.

2. Walcarius A, Mercier L (2010) Mesoporous organosilica adsorbents: nanoengineered materials for removal of organic and inorganic pollutants. J Mater Chem 20: 4478-4511.

3. Jal PK, Patel S, Mishra B (2004) Chemical modification of silica surface by immobilization of functional groups for extractive concentration of metal ions. Talanta 62(5): 1005-1028.

4. Daniels DR, Donald AM (2004) Soft Material Characterization of the Lamellar Properties of Starch: Smectic Side-Chain Liquid-Crystalline Polymeric Approach 37: 1312-1318.

5. White RJ, Budarin VL, Clark JH (2008) Tuneable mesoporous materials from alpha-D-polysaccharides. Chem Sus Chem 1: 408-411.

6. He H, Yang S, Yu K, Ju Y, Sun C, et al. (2010) Microwave induced catalytic degradation of crystal violet in nano-nickel dioxide suspensions. J Hazard Mater 173(1-3): 393-400.

7. Chen Z, Deng M, Chen Y, He G, Wu M, et al. (2004) Preparation and performance of cellulose acetate/polyethyleneimine blend microfiltration membranes and their applications. J Memb Sci 235(1-2): 73-86.

8. Chindaprasirt P, Rukzon S (2008) Strength, porosity and corrosion resistance of ternary blend Portland cement, rice husk ash and fly ash mortar. Constr Build Mater 22(8): 1601-1606.

9. Chuah T, Jumasiah A, Azni I, Katayon S, Thomas Choong S (2005) Rice husk as a potentially low cost biosorbent for heavy metal and dye removal: an overview. Desalination 175: 305-316.

\section{Your next submission with Juniper Publishers will reach you the below assets}

- Quality Editorial service

- Swift Peer Review

- Reprints availability

- E-prints Service

- Manuscript Podcast for convenient understanding

- Global attainment for your research

- Manuscript accessibility in different formats

( Pdf, E-pub, Full Text, Audio)

- Unceasing customer service

Track the below URL for one-step submission https://juniperpublishers.com/online-submission.php 